\title{
Hybrid gels: Influence of oleogel/hydrogel ratio on rheological and textural properties
}

\author{
Artur J. Martins ${ }^{\mathrm{a}, \mathrm{b}, *}$, Pedro Silva ${ }^{\mathrm{a}}$, Filipe Maciel $^{\mathrm{a}}$, Lorenzo M. Pastrana ${ }^{\mathrm{b}}$, Rosiane Lopes Cunha ${ }^{\mathrm{c}}$, \\ Miguel A. Cerqueira ${ }^{\mathrm{b}}$, António A. Vicente ${ }^{\mathrm{a}}$ \\ ${ }^{\text {a }}$ Centre of Biological Engineering, University of Minho, Campus de Gualtar, 4710-057 Braga, Portugal \\ ${ }^{\mathrm{b}}$ International Iberian Nanotechnology Laboratory, Av. Mestre José Veiga s/n, 4715-330 Braga, Portugal \\ ${ }^{\mathrm{c}}$ Department of Food Engineering, Faculty of Food Engineering, University of Campinas, UNICAMP, CEP: 13083-862 Campinas, SP, Brazil
}

\section{A R T I C L E IN F O}

\section{Keywords:}

Hybrid gelation

Texture

Rheology

Wax

Polysaccharide

\begin{abstract}
A B S T R A C T
Hybrid gels can be used for controlled delivery of bioactives and for textural and rheological modification of foods. In this regard the hydrogel:oleogel ratio and gel development methodologies showed to be the aspects that influence most of their properties. The present study shows how different fractions of oleogel can influence the hydrogel matrix of an oleogel-in-hydrogel emulsified system in terms of polymorphic arrangement, microstructure, texture and rheology. The hydrogel was prepared by using an aqueous sodium alginate solution and the oleogel was prepared through the gelation of medium chain triglycerides with beeswax. Hybrid gels were prepared under constant shearing. Crystallinity was clearly changed as hydrogel and oleogel were combined. No polymorphism was observed in the X-Ray diffraction of hybrid gels, as these showed homogeneous results for all component ratios. The behaviour of samples with increasing oleogel-to-hydrogel ratio presented a decrease of both firmness and spreadability, and then, a decrease of gel adhesivity and cohesiveness. This textural response was a consequence of the disaggregated structure, stemming from the disruption of the hydrogel network, due to the inclusion of increasing amounts of oleogel. Rheological results showed that all hybrid gels presented a gellike behaviour $\left(G^{\prime}>G^{\prime}\right)$. Oleogel's strength influenced the overall textural and rheological performance of hybrid gels. This work demonstrates the possibility of producing hybrid gels aiming to tailor texture on food systems.
\end{abstract}

\section{Introduction}

Hydrogels present extremely interesting properties (i.e. easy spreadability, easy to clean, water-solubility; miscibility, nonstaining) and their compatibility with a wide range of excipients (i.e. solvents), allows using them in a wide range of applications (Peppas et al., 2000). Pharmaceutical and tissue engineering applications are some examples that display a number of user compliance features of hydrogels (e.g. non-oily nature, swelling behaviour and cooling effect and the ability to be simply removed from the body just using water) (Peppas et al., 2000). Biodegradable and biomimetic properties associated to hydrogels showed to be relevant when they are used for development of innovative drug delivery systems (Lin and Metters, 2006; Peppas et al., 2000). Some drawbacks are associated to these structures, when considering their use as vehicles of bioactive substances through the lipophilic barrier of the skin (Mura et al., 2000). The difficulty to solubilize lipophilic compounds under these circumstances has been a starting point to further developments that consider the elaboration of particulate hydrogel microspheres or complex hydrogels, composed by small oil droplets entrapped within a biopolymer or protein-coated fat droplets, aiming the delivery of lipophilic bioactive compounds through the skin barrier (Matalanis and McClements, 2013; Mun et al., 2015). In terms of food applications, some interesting developments have been reported in recent years, like oil-filled caseinate-rich hydrogel particles (Chung et al., 2013) and chemically stable curcumin through filled alginate hydrogel beads (Zheng et al., 2017). Soradech and co-workers showed very promising results using an emulsified gel consisting of different waxes inside an alginate-based gel bead aiming at controlled tamarind seed extracts release (Soradech et al., 2016). Nevertheless, direct applications of hydrogels in foods, as replacement for other ingredients, is still a challenge.

Difficulties in mimicking structural and textural properties of foods are a concern. Emulgels were pointed as one of the solutions to overcome some of the above-mentioned shortcomings attributed to

\footnotetext{
* Corresponding author at: Centre of Biological Engineering, University of Minho, Campus de Gualtar, 4710-057 Braga, Portugal.

E-mail address: arturmartins.web@gmail.com (A.J. Martins).
} 
hydrogels. Emulgels result from an emulsification (oil-in-water or water-in-oil) where the system will be gelled by means of cross-linking between the chains of the compounds present in the mixture; e.g. stearate organogel-gelatin bigels (Sagiri et al., 2015) and and sequential charged nano-emulsions (Soltani and Madadlou, 2016). The amphiphilic functional behaviour, promoted by both hydrophilic and lipophilic affinities of their constituents, potentiates the usage of emulgels towards the delivery of bioactive compounds. Nonetheless, emulgels have been less appreciated by consumers due to their pastiness/stickiness, and phase separation (Sagiri et al., 2015). In terms of pharmaceuticals, some of these drawbacks are pointed to oleogels too. Oleogels (or organogels) are soft matter structures, that are able to structure oils as a result of an entangled network that is formed during the cooling stage of development. Oleogels can be produced using different types of oils in which they can transport lipophilic compounds for pharmaceutical and food applications (Patel and Dewettinck, 2016; Wang et al., 2016). Great attention from the scientific and industrial communities has been directed towards oleogels once they have been mentioned as one of the main answers to act as fat replacers, offering a healthier product while presenting a tailor-made performance (Ergun et al., 2016; Martins et al., 2018; Mert and Demirkesen, 2016; Rogers et al., 2014; Sloan, 2015; Utrilla et al., 2014). One of the most interesting features is their ability to present gel-like behaviour even using low amounts of gelator molecules (e.g. beeswax; ethyl-cellulose; fatty acid derivatives; oryzanol combined with phytosterols) (Cerqueira et al., 2017; Martins et al., 2016; Öğütcü and Y1lmaz, 2014; Patel, 2015). Previous works reporting on beeswax-based oleogels, demonstrated their ability to structure different types of oil and to incorporate bioactive compounds (Martins et al., 2016; Patel and Dewettinck, 2016).

The combination of hydrogels and oleogels results in the so-called hybrid gels (or bigels), with both hydrophilic and lipophilic characteristics. Hybrid gels are biphasic systems that conjugate water-based gels (hydrogels) and oil-based gels (oleogels). Hybrid gels application in food products must be focused towards their implementation in complex food systems, in similar ways as hydrogels and oleogels. Unique thermodynamic behavior, viscoelasticity and the variety of materials that can be used are among the most important characteristics of this type of gels. These properties can also be tuned with formulation adjustments and material combinations that increase their potential (Cakmakc1 Gundogdu and Kavaz, 2008; Esposito et al., 2018; Lupi et al., 2015; Singh et al., 2014). In fact, such applications of hybrid gels can target major developments for food industry that will represent an alternative for the enrichment of food nutritional. These systems have been studied regarding their capacity for controlled release of active compounds like omega-3 fatty acids (Rehman et al., 2014) and ciprofloxacin (Sagiri et al., 2015) and metronidazole (Wakhet et al., 2015). Our objectives with the present work were to improve the knowledge and expand hybrid gels' applications. We reported on the hybrid gels' textural and rheological behaviour aiming at their tailoring capabilities for further use in food applications. Therefore, we studied the incorporation of a beeswax-based oleogel (produced with different oleogelator concentrations, 3 and $6 \% \mathrm{w} / \mathrm{w}$ ) into a sodium alginate hydrogel and how this would influence the resulting hybrid gel' structural properties at nano-, micro- and macroscale level.

\section{Materials and methods}

\subsection{Raw materials and hybrid gel preparation}

Three control samples were produced, namely two samples of oleogel (with increasing oleogelator amount) and one hydrogel. Oleogels were prepared using beeswax, as the oleogelator and medium chain triglycerides (MCT) Neobee with 55\% C7H15 and 44\% C9H19 as the nonpolar solvent. Beeswax was kindly offered by Poth Hille \& Co Ltd. (Rainham, UK) and the MCT was gently donated by Stepan
Company (Northfield, USA). Based on previous research reports performed by our group, the oleogels (OG) were: OG3 (with $3 \% w / w$ of oleogelator) and OG6 (with $6 \% \mathrm{w} / \mathrm{w}$ of oleogelator); these were produced by dissolving beeswax under agitation at $80^{\circ} \mathrm{C}$ in MCT oil until full solubilization during approximately $30 \mathrm{~min}$ (Martins et al., 2016). Control samples (alginate hydrogel and beeswax oleogels) were submitted to the same shearing procedure. Subsequently, the mixture was left at room temperature (approx. $23^{\circ} \mathrm{C}$ ), during at least $2 \mathrm{~h}$ to achieve oleogelation. In the case of the hydrogel (AL), only one type (with constant sodium alginate concentration) was produced. The sodium alginate hydrogel $(2 \% \mathrm{w} / \mathrm{w})$ was produced at room temperature using sodium alginate from FMC Corporation (Philadelphia, USA), with 35/ $65 \mathrm{G} / \mathrm{M}$ ratio (1,4-linked alpha-L-guluronic acid (G) and beta-D-mannuronic acid (M) units) that was kindly offered by Eurosalmo Ltd. (Matosinhos, Portugal). Sodium alginate was poured in distilled water under strong and constant stirring at room temperature until full solubilization. We chose the concentration of $2 \%(w / w)$ for sodium alginate because it revealed to be appropriate to develop a gel-like structure after full solubilization without heating.

Hybrid gels (HGs) were produced by mixing the oleogel (at room temperature $22 \pm 2{ }^{\circ} \mathrm{C}$ ) after complete gelation, with the hydrogel (alginate solution described above) in different ratios, using a helical mechanical mixer at $600 \mathrm{rpm}$ during $45 \mathrm{~min}$. Samples were then stored at $4{ }^{\circ} \mathrm{C}$, stabilized at room temperature during $24 \mathrm{~h}$ before being analysed. In total, samples with 5 different ratios hydrogel:oleogel (1:99; $5: 95 ; 10: 90 ; 20: 80 ; 50: 50)$ were developed: HG3-1:99; HG3-5:95; HG3-10:90; HG3-20:80 and HG3-50:50 (using OG3) and HG6-1:99; HG6-5:95; HG6-10:90; HG6-20:80 and HG6-50:50 (using OG6). Every sample was tested for fluency with the test tube tilting method.

\subsection{X-ray diffraction}

X-Ray Diffractometer X Pert PRO MRD system from Malvern Panalytical Ltd. (Royston, UK), was used to execute X-ray diffraction analysis (XRD) with the finality to study the crystalline polymorphism among HG samples. The X-ray diffraction data were acquired at room temperature, using angular scans from $5.0^{\circ}$ to $50^{\circ}(2 \theta)$, performed with a Cu source, X-ray tube $(\lambda=1.54056 \AA)$ at $45 \mathrm{kV}$ and $40 \mathrm{~mA}$. The fine calibration offset for $2 \theta=-0.0372^{\circ}$. Information was collected during $174 \mathrm{~s}$ and PANanalytical X'Pert HighScore Plus software was used to gather data and the analysis of peak diffractions. Determination of the lattice parameter $d$ was performed using Bragg's law, where $\lambda$ is the wavelength of the X-ray used, $\theta$ is the half of the diffraction Bragg angle (20) and $d$ is the space between planes.

$n \lambda=2 d \sin \theta$

\subsection{Bright-field and fluorescence microscopy}

Bright field micrographs were obtained with a Nikon Instruments Wide-Field Upright Ni-E Microscope (Tokyo, Japan) equipped with a Nikon digital camera, supported by the NIS-Elements Microscope Imaging Software, also from Nikon. The samples were prepared after hybrid gels settling at room temperature. After that, the small samples were cut and placed carefully on the glass support with a cover glass and then observed under the microscope. Extreme care was taken to avoid influencing the gel's structure (e.g., the cover glass was simply laid over the gel and no pressure was exerted on the sample). A fluorescence microscope Olympus BX51 (Tokyo, Japan) was used to understand the structural arrangement of the oleogel particles in hybrid gel samples.

\subsection{Textural measurements}

Three parameters (firmness, spreadability, and adhesivity) were chosen for texture measurements and performed using a double axis 

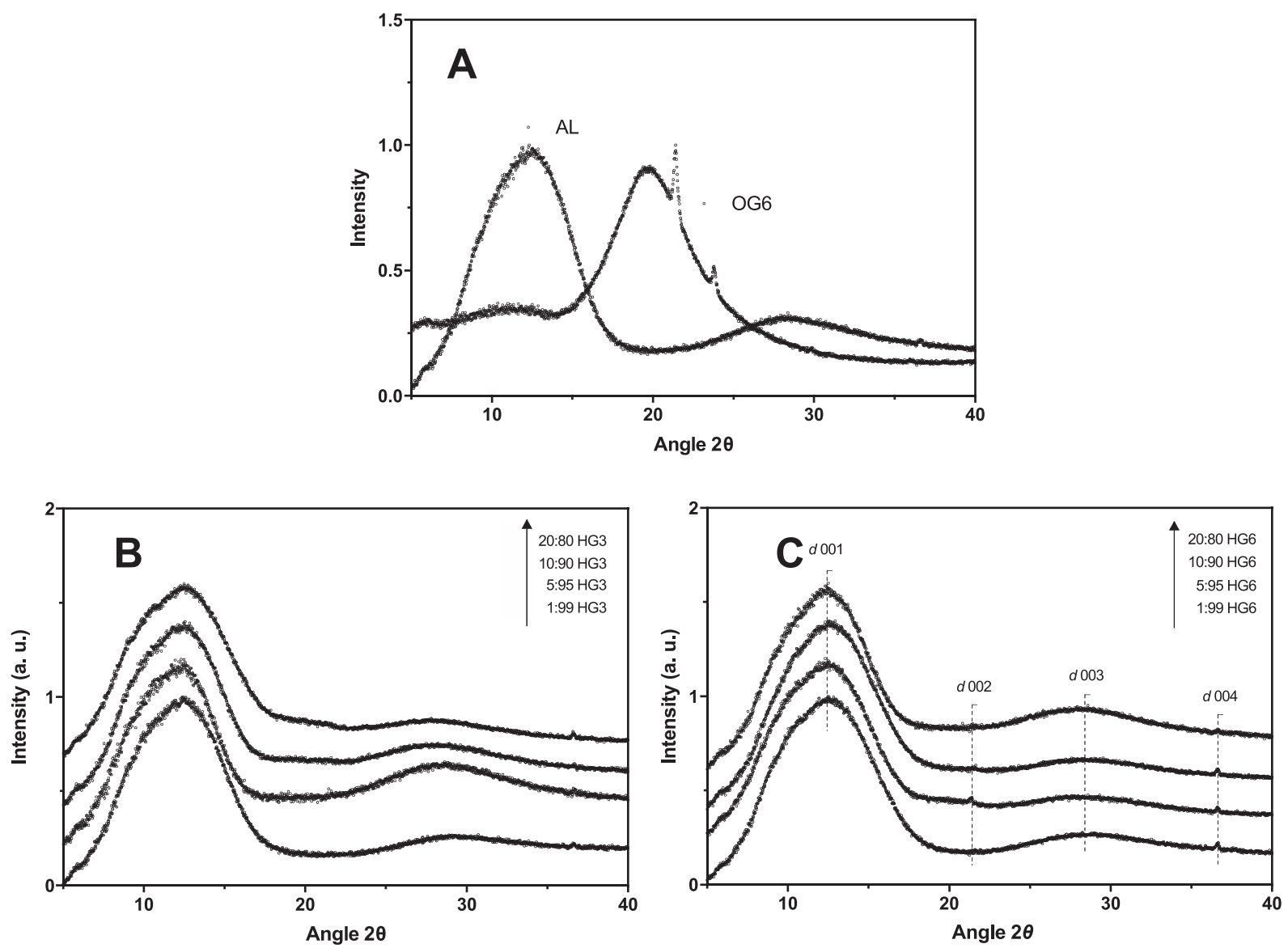

Fig. 1. XRD patterns for A) Control samples; B) and C) hybrid gels with OG3 and OG6 respectively.

texture analyzer TA.HD PLUS from Stable (Surrey, UK) using a load cell of $5 \mathrm{~kg}$ with a conical TTC Spreadability Rig (HDP/SR) attachment (Stable Microsystems, Surrey, UK) consisting of a set of precisely matched male (positive) and female (negative) acrylic $90^{\circ}$ cones. The test involved traveling $24 \mathrm{~mm}$ from a fixed position $25 \mathrm{~mm}$ over the bottom of the lower cone. Four samples from each formulation were evaluated at room temperature. The firmness, spreadability (maximum force and work of shear respectively) and adhesivity data were obtained and analysed. Regarding data collection, the maximum force and work of shear were extracted from the force vs time curve and related as indicators of product firmness and spreadability, respectively (Sanders Iii et al., 2014). The peak force value is related to the maximum compression force (uniaxial), designated as "firmness" at the depth specified in the test. Because of the continuous structural network that forms the gels, higher firmness values translate to a larger area under the force vs time curve, corresponding to a higher total amount of force required to perform the shearing process; this is designated as spreadability. In the last step of the textural test, the conical probe withdraws/ leaves the sample and any adhesivity demonstrated by a negative force region on the shown curve is recorded, which is related with the work of adhesion (Kamboj and Rana, 2014). This conical spreadability rig test provides a more complementary understanding about the materials/gels rather than compression test alone.

Before testing, the positive cone probe was calibrated against the negative cone so that the starting point is at the same height for each test ( $25.0 \mathrm{~mm}$ above the female cone). The software is used to calibrate the probe with a return distance of $25.0 \mathrm{~mm}$, after raising the positive cone. The probe travelled downwards, touched the negative cone, and then returned to a position precisely $25.0 \mathrm{~mm}$ over the calibrating point. The hybrid gel was filled into the negative cone, allowed to equilibrate at room temperature overnight and then positioned in the base holder for testing. Spreadability was obtained from the area under the curve force vs time, and adhesiveness was gathered from the negative values of this curve. Each treatment was performed in triplicate. For data analysis, software Texture Exponent ver. 6.1.1.0 by Stable Microsystems (Surrey, UK) was used.

\subsection{Rheology}

All rheological measurements were made using a Discovery Hybrid Rheometer (DHR1) from TA Instruments (New Castle, USA) with Peltier temperature set to $25^{\circ} \mathrm{C}$. TRIOS Software was used to control the equipment and to acquire rheological parameters. The hybrid gels were tested after the setting period. Small samples were cut and placed carefully on the surface of the rheometer Peltier plate. A stainless-steel cone-plate geometry of $60 \mathrm{~mm}$, with an angle of $2.006^{\circ}$ and truncation of $64 \mu \mathrm{m}$, was used due to its capability of generate uniform shear rate across the entire gel sample. Regarding oscillatory rheometry measurements, the viscoelastic properties of HG were assessed by utilizing a frequency sweep range between 0.01 and $30 \mathrm{~Hz}$. This test was performed to evaluate the material response, at a constant strain amplitude of $1 \%$ for the whole range of deformation frequencies (and within the region of reversible deformation). Flow curves were also used in order to understand the behaviour of HGs when large rates of deformation are applied. This test is important to fully understand the behaviour of the different gel matrices under shear growth. Three replicates of each HG sample were recorded for every test.

\subsection{Statistical analysis}

The experiments subjected to statistical analysis were carried out in triplicate. The statistical analyses were performed using analysis of 
variance, Tukey's mean comparison test $(p<.05)$ from results conveyed as average and standard deviation (GraphPad Software Inc., San Diego, USA).

\section{Results and discussion}

\subsection{X-ray diffraction pattern of hybrid gels}

Fig. 1A shows the diffraction pattern for AL and OG6 controls and Figs. 1B and 1C show HG3 and HG6 samples, respectively (it was not possible to measure OG3 due to its runny consistency and therefore no self-sustainment in the XRD sample support). Fig. 1B and C showed the XRD patterns of the HG samples in order to better understand the differences. For each HG the recorded diffraction pattern was very similar to the pattern recorded for the AL control. Results allow concluding that as long as the HGs are formed, the absence of polymorphism is reached. Polycrystallinity is detected for OG6 (Fig. 1A) in wide angle regions with $d$-spacings in the range of 3.74 to $8.04 \AA$. AL control exhibited a broad peak around $13^{\circ}$ that is attributed to the presence of an amorphous region in the polymer (Li et al., 2012). This peak does not present big changes after the incorporation of oleogel, however, a little variation was visible in some peaks for HG3 samples between $10^{\circ}$ and $12.5^{\circ}$. Results also show a very dim intensity change around $21.5^{\circ}$, in this case these reflections are more accentuated in HG6. The mentioned alterations in diffraction are possibly a result of the introduction of the oleogel (beeswax) that is responsible for a more ordered structural arrangement. When the HG are produced the crystalline arrangement remains consistent, as larger quantities of crystalline particles are introduced in the hybrid gel system (i.e. higher oleogel fraction).

\subsection{Morphological and microscopy analyses}

Fig. 2 provides information on the stability of controls and HG, as the tilted tubes showed that OG3 is not self-standing while OG6 presents a gel-like behaviour. This behaviour is a consequence of the hybrid gels' properties produced with higher OG amounts. In the case of

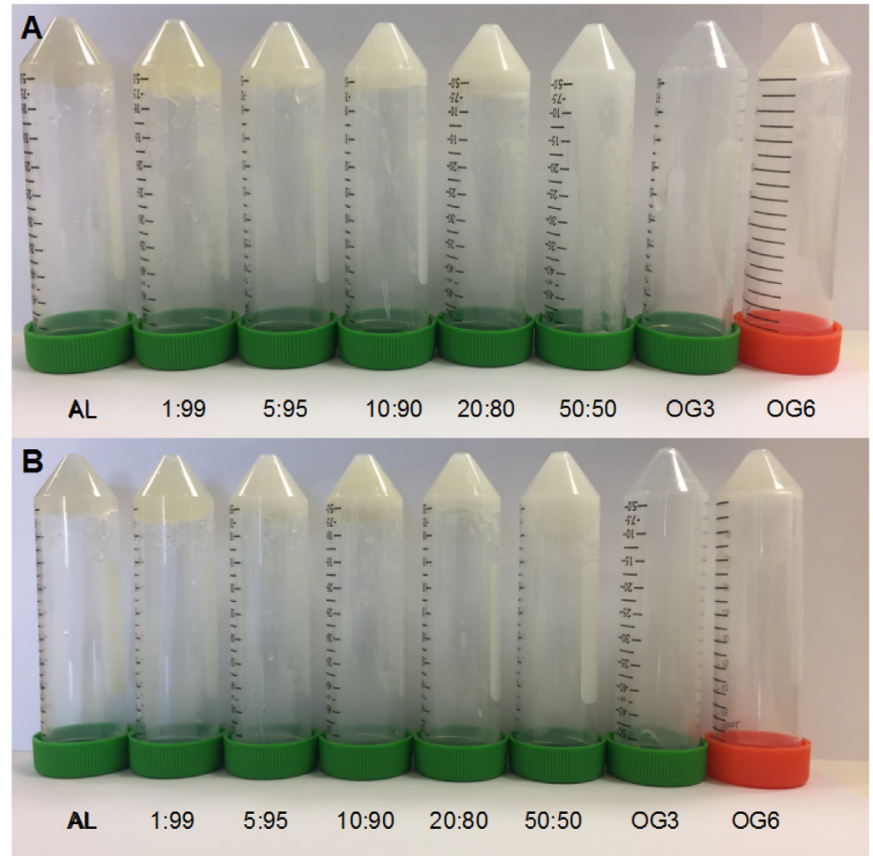

Fig. 2. Structural behaviour of hybrid gels at different ratios (1:99, 5:95, 10:90, $20: 80,50: 50$ ) (A) Hydrogel control (AL), hybrid gels with OG $3 \%(w / w)$ and oleogel controls (OG3 and OG6). (B) Hydrogel control (AL), hybrid gels with OG $6 \%(w / w)$ and oleogel controls.
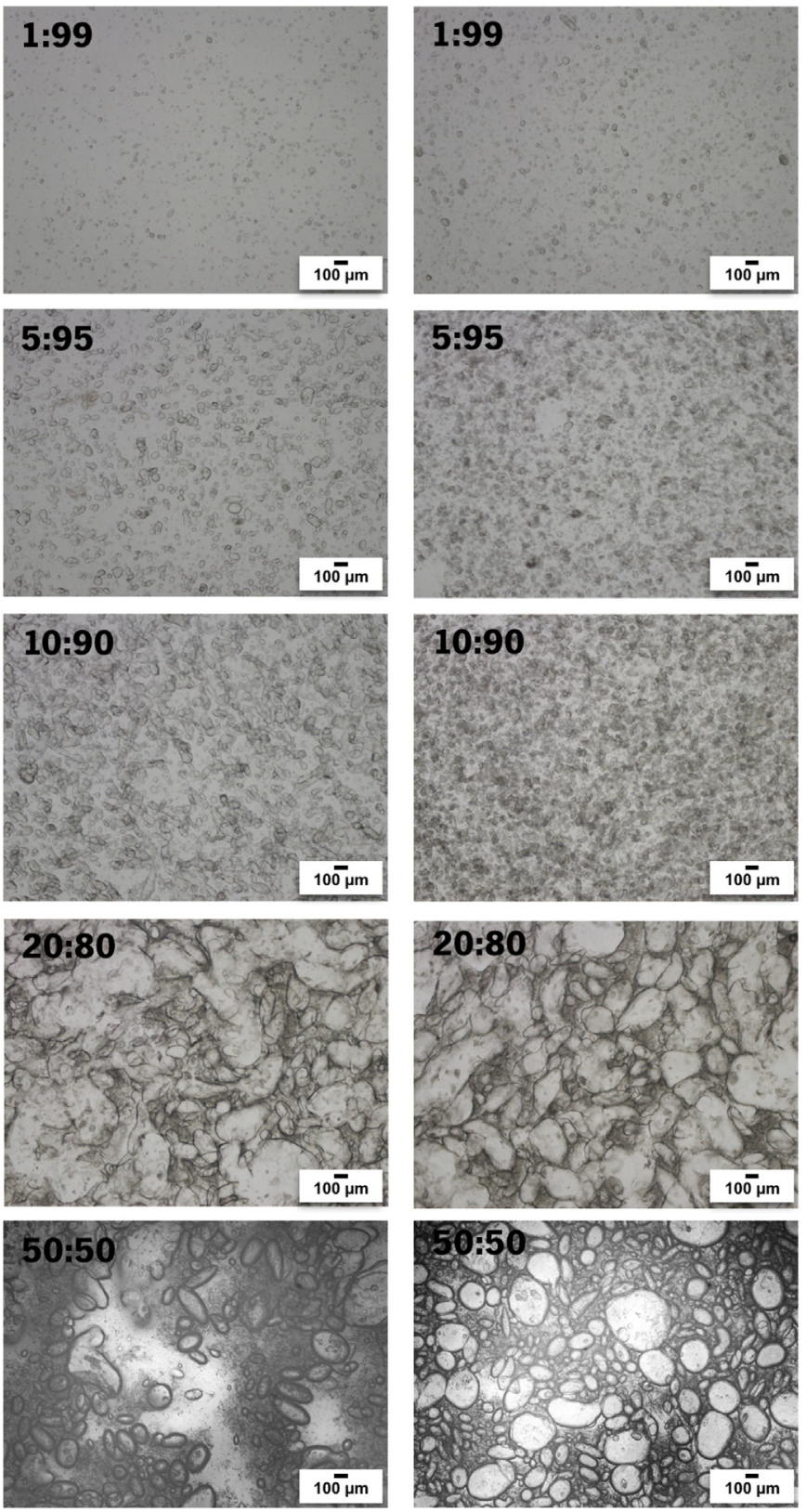

Fig. 3. Micrographs of hybrid gels with the increasing of oleogel ratio. Column in the left presents hybrid gels with OG 3\% (w/w); and column in the right presents hybrid gels with OG $6 \%(\mathrm{w} / \mathrm{w})$. Micrographs obtained with a magnification of $15 \times$.

HG3 50:50 the sample flows as the tube is tilted, while in the case of HG6-50:50 the sample remained self-standing. This behaviour can be explained by the properties of the OG and their crystal network, that is arranged in lamellar conformation that is formed during the cooling process of oleogel formation (Martins et al., 2016). The shearing process during hybrid gel production is responsible for less ordered crystalline oleogel particles. For HG3 the oleogel particles were not distributed in the same way as for HG6, therefore presenting a runnier (liquid) consistency in some ratios. For HG3 samples, a more heterogeneous distribution of oleogel (elongated particles with larger sizes, namely in smaller oleogel ratios), thus conveying less structural strength and thus explaining the flow of the gel. However, it is important to notice that the shear process during gels mixing is responsible for the incorporation of oleogel inside the hydrogel matrix, leading to the oleogel-in-hydrogel structural arrangement. Concerning 
Table 1

Firmness, spreadability and adhesivity of gel samples.

\begin{tabular}{|c|c|c|c|c|}
\hline Samples & & Firmness (N) & Spreadability (N.s $\left.{ }^{-1}\right)$ & Adhesivity (N.s ${ }^{-1}$ ) \\
\hline \multirow[t]{3}{*}{ Controls } & $\mathrm{AL}$ & $12.03 \pm 0.64^{\mathrm{a}}$ & $14.14 \pm 1.05^{\mathrm{a}}$ & $-2.88 \pm 0.33^{\mathrm{a}}$ \\
\hline & OG3 & $0.213 \pm 0.001^{\mathrm{e}, \mathrm{H}}$ & $0.082 \pm 0.002^{\mathrm{e}, \mathrm{H}}$ & $-0.037 \pm 0.002^{\mathrm{c}, \mathrm{H}}$ \\
\hline & OG6 & $0.39 \pm 0.02^{\mathrm{f}, \mathrm{I}}$ & $0.25 \pm 0.01^{\mathrm{d}, \mathrm{I}}$ & $-0.073 \pm 0.003^{\mathrm{e}, \mathrm{I}}$ \\
\hline \multirow[t]{5}{*}{ HG3 } & $1: 99$ & $12.60 \pm 0.60^{\mathrm{a}, \mathrm{A}}$ & $14.66 \pm 0.54^{\mathrm{a}, \mathrm{A}}$ & $-3.11 \pm 0.27^{\mathrm{a}, \mathrm{b}, \mathrm{A}}$ \\
\hline & $5: 95$ & $9.12 \pm 0.23^{\mathrm{b}, \mathrm{A}}$ & $11.95 \pm 0.35^{\mathrm{b}, \mathrm{A}}$ & $-2.44 \pm 0.14^{\mathrm{a}, \mathrm{A}}$ \\
\hline & $10: 90$ & $5.59 \pm 0.29^{\mathrm{c}, \mathrm{B}}$ & $8.02 \pm 0.61^{\mathrm{c}, \mathrm{B}}$ & $-0.88 \pm 0.10^{\mathrm{c}, \mathrm{B}}$ \\
\hline & $20: 80$ & $2.62 \pm 0.07^{\mathrm{d}, \mathrm{D}}$ & $2.99 \pm 0.01^{\mathrm{d}, \mathrm{D}}$ & $-0.71 \pm 0.01^{\mathrm{c}, \mathrm{d}, \mathrm{D}}$ \\
\hline & $50: 50$ & $0.57 \pm 0.01^{\mathrm{e}, \mathrm{F}}$ & $0.52 \pm 0.02^{\mathrm{e}, \mathrm{F}}$ & $-0.13 \pm 0.00^{\mathrm{d}, \mathrm{e}, \mathrm{F}}$ \\
\hline \multirow[t]{5}{*}{ HG6 } & $1: 99$ & $11.95 \pm 0.87^{\mathrm{a}, \mathrm{A}}$ & $14.14 \pm 0.96^{\mathrm{a}, \mathrm{A}}$ & $-2.49 \pm 0.00^{\mathrm{a}, \mathrm{A}}$ \\
\hline & 5:95 & $9.06 \pm 0.38^{\mathrm{b}, \mathrm{A}}$ & $12.22 \pm 0.85^{\mathrm{a}, \mathrm{A}}$ & $-2.81 \pm 0.15^{\mathrm{a}, \mathrm{A}}$ \\
\hline & 10:90 & $6.47 \pm 0.12^{\mathrm{c}, \mathrm{C}}$ & $9.64 \pm 0.29^{\mathrm{b}, \mathrm{C}}$ & $-1.60 \pm 0.30^{\mathrm{b}, \mathrm{C}}$ \\
\hline & $20: 80$ & $3.07 \pm 0.02^{\mathrm{d}, \mathrm{E}}$ & $3.23 \pm 0.12^{\mathrm{c}, \mathrm{E}}$ & $-0.82 \pm 0.01^{\mathrm{c}, \mathrm{E}}$ \\
\hline & $50: 50$ & $1.52 \pm 0.01^{\mathrm{f}, \mathrm{G}}$ & $1.37 \pm 0.05^{\mathrm{c}, \mathrm{d}, \mathrm{G}}$ & $-0.33 \pm 0.01^{\mathrm{c}, \mathrm{d}, \mathrm{G}}$ \\
\hline
\end{tabular}

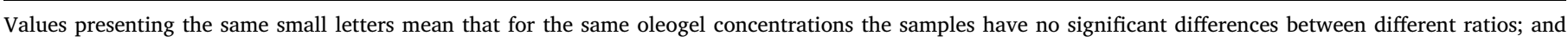
values presenting the same capital letters mean that for the same ratio the samples have no significant difference between different oleogelator concentrations.
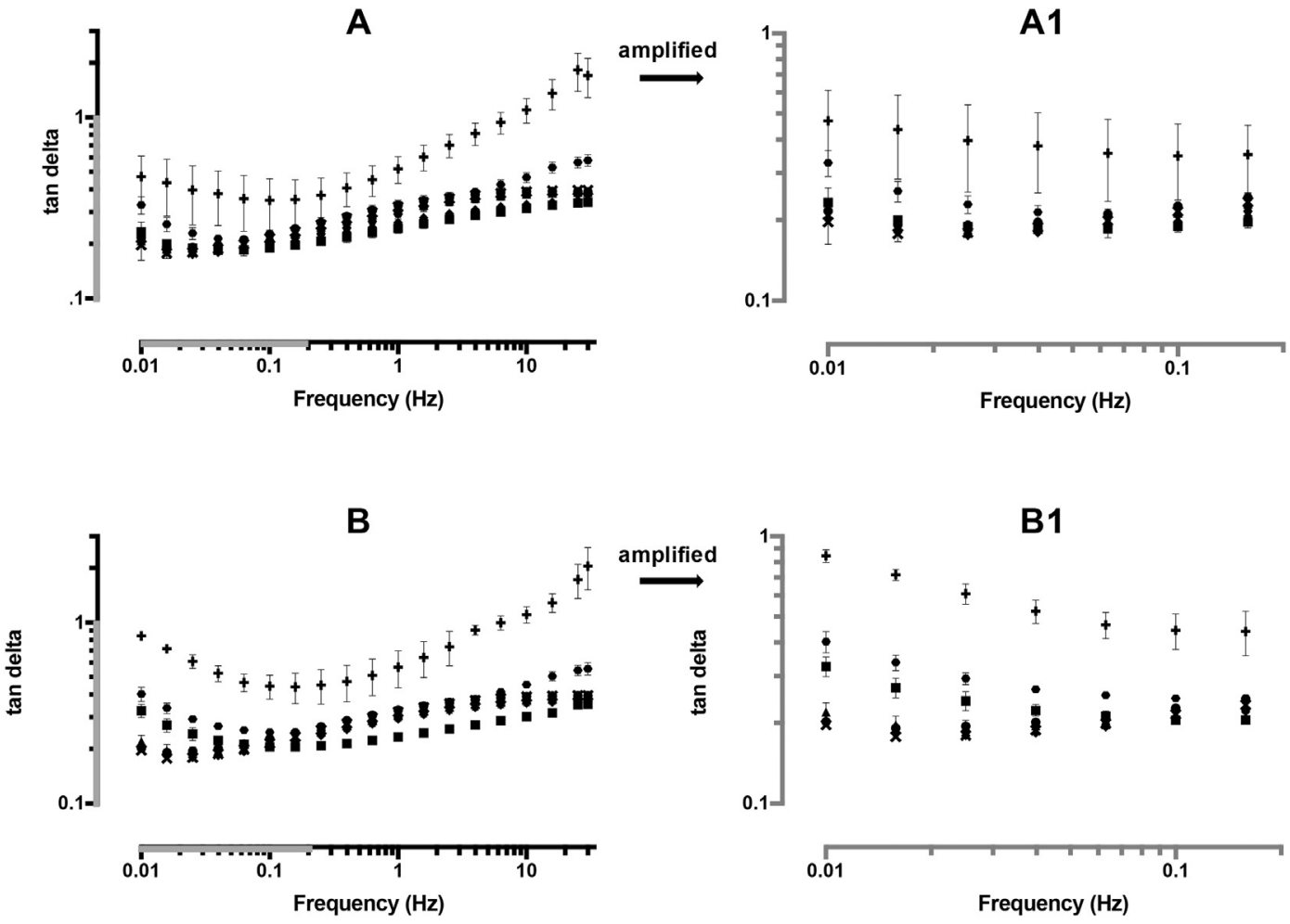

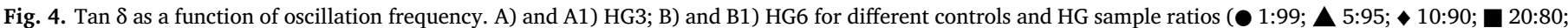
50:50; + control OG; × control AL). A1 and B1 represent a "zoomed in" section of the tested frequency range.

the HG samples with the same amount of oleogel, the differences regarding oleogel particles distribution within the hydrogel matrix are quite clear once alongside with the increasing oleogel incorporation ratio a globular gelled structure is increasingly more heterogeneous (Fig. 3). The imprisoning of oil droplets in the structure is confirmed by fluorescence microscopy images (see Fig. SM1) and the stability test performed by centrifugation provided information about the increased binding capacity of HG6 as compared to HG3 samples (see Fig. SM2).

\subsection{Textural measurements}

Texture measurements provided useful information on how the oleogel concentration and different oleogel incorporation influenced the HGs textural parameters, such as firmness, spreadability and adhesivity.

Results showed that higher gelator's concentration (HG3 and HG6) only influences significantly the textural behaviour of HG samples (Table 1) after reaching a ratio of 10:90. Regarding the influence of the ratio (for HG3 and HG6 separately) it was verified the decrease of firmness and spreadability values along with the increase of oleogel fraction in the overall composition of hybrid gels. This decrease is statistically significant in the majority of the samples (with the same gelator concentration). The exception was for HG samples with closer composition to the controls, namely control AL with 1:99 and 50:50 with oleogel control samples (OG3 and OG6), in which no statistically significant differences were observed.

The mixing process of both gel constituents is responsible for the introduction of dispersed oleogel particles inside the hydrogel matrix. This incorporation is seen in Fig. 3 and Fig. SM1 (supplementary material) where structures with oleogels as the disperse phase and hydrogel as the continuous phase can be observed. When the oleogel amount increases in the overall composition of HGs (i.e. high OG ratio), 


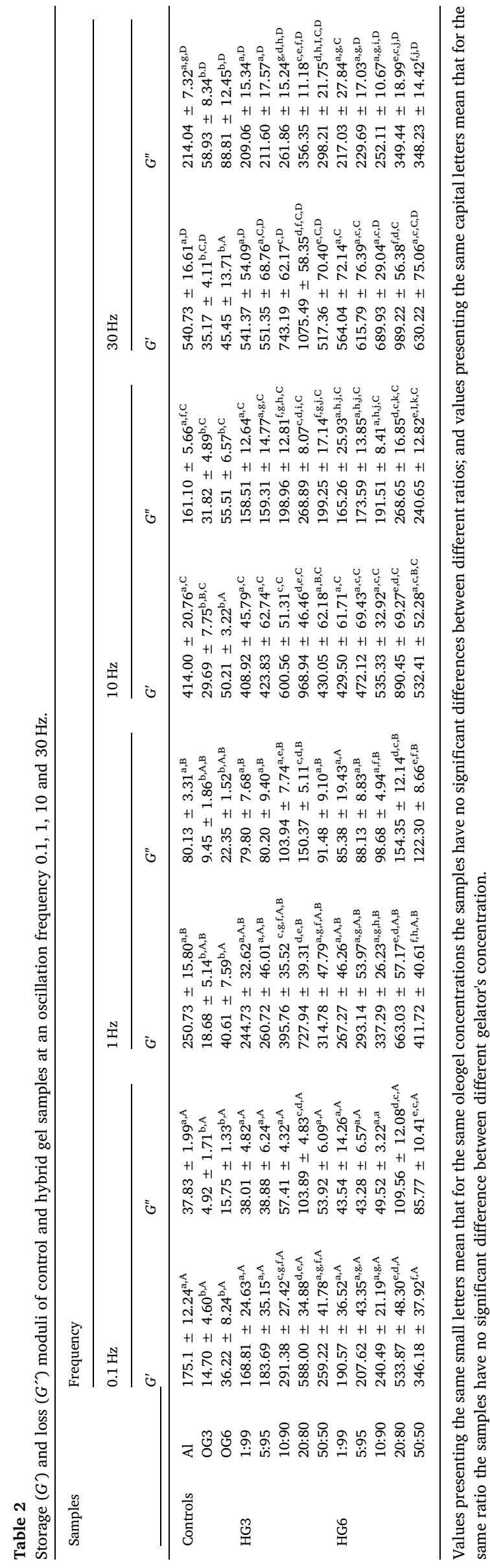

the consistency of the gel changes as the particle arrangements are also modified, as seen in Fig. 3. The addition of the oleogel, weakens the gel structure promoting a more disaggregated structure that will flow easily and provide less resistance for the textural probe. It should also be taken into account that an extrusion factor is also associated to this test: as the conical probe is penetrating the sample, the samples with higher oleogel fraction will be extruded with less opposition or contrary force, and that behaviour is translated to the spreadability of the gels. The adhesivity profile of HGs is an indication of the response of the material and its consequent resistance to separate from the cone base. The more stickiest the sample is, the more negative the value will be; the influence of hydrogel and oleogel fractions in this parameter is well observable in the adhesivity data presented in Table 1 , where is possible to understand that a larger disaggregated gel (with increased oleogel mass) reveals a less adhesive textural response.

\subsection{Rheological analysis}

Low deformation oscillatory rheology was performed in HG3 and HG6 samples in order to understand the rheological behaviour of hybrid gels using various hydrogel/oleogel ratios. The effects associated to the progressive increase of the oleogel ratio led to a decrease in the integrity of the generated gel matrix, in a way that these gels could flow easily. Based on the microstructure of the HGs, it may be concluded that the increase of the size of oleogel particles and the consequent possible phase separation in HGs structure can explain this behaviour. Fig. 4 shows the $\tan \delta$ values that were obtained for a range of selected points of applied frequencies. The gathered data shows the reduced values that HGs demonstrate, evidencing their solid-like behaviour, in contrast to both oleogel control samples (OG3 and OG6). These results reveal a transition that occurs in the oleogel control, from solid-like to a liquidlike performance. This is translated by the increase of $\tan \delta$ values $(\tan \delta>1)$ when frequency application is amplified. This trend is also observed for AL (hydrogel control), however this remains solid-like for all the frequency range. Approx. 50 rads/s (close to $10 \mathrm{~Hz}$ ) can be used as the value of human perception of thickness (Hill et al., 1995). Fig. 4 shows this tendency for the samples produced with the 20:80 ratio that presented lower $\tan \delta$ values (more solid-like or elastic prevailing) than the other HG samples as frequency is increased. The close-up shows this cross-over (Fig. A1 and Fig. B1). On the other hand the OG controls showed a more liquid-like behaviour. Despite from this, no major differences are observed demonstrating major influence of using OG6 versus OG3 in hybrid gel formulation.

Both HG3 and HG6 can be classified as a gel since $G^{\prime}$ is generally an order of magnitude larger than $G^{\prime \prime}$ (below $10 \mathrm{~Hz}$ ), which can be observed in Table 2. The storage and loss moduli information regarding gels' stress response in oscillatory shear, demonstrated that for HGs there was no cross-over, this is a typical behaviour of elastic networks which means that the mixture of oleogel and hydrogel produced HGs with a solid-like behaviour (Yan and Pochan, 2010). In contrast, oleogel control samples, demonstrated to be frequency dependent, as the crossover between the elastic and the viscous moduli occurred at higher frequencies. Samples with a 20:80 ratio for HG3 and HG6 revealed the highest value of storage modulus $(G)$ in the studied frequency range among all HGs. These samples are accountable for a more pronounced response due to a higher interaction between the big oleogels particles inside the alginate polymeric matrix (Fig. 3). This might have happened, in contrast to the 50:50 samples which had an even higher oleogel fraction, because the oleogel concentration (20:80) is not sufficiently high, to promote a disaggregated gel and phase separation. In the sample HG 20:80 the hydrogel matrix was maintained in the outer side, while for the HG 50:50 was in part less organized, being the oleogel not fully incorporated in the hydrogel and exposed to the outside of the hydrogel matrix. In comparison to the hydrogel control, this was observed in all HG samples as a response to the increase of oleogel particles in gel composition. 

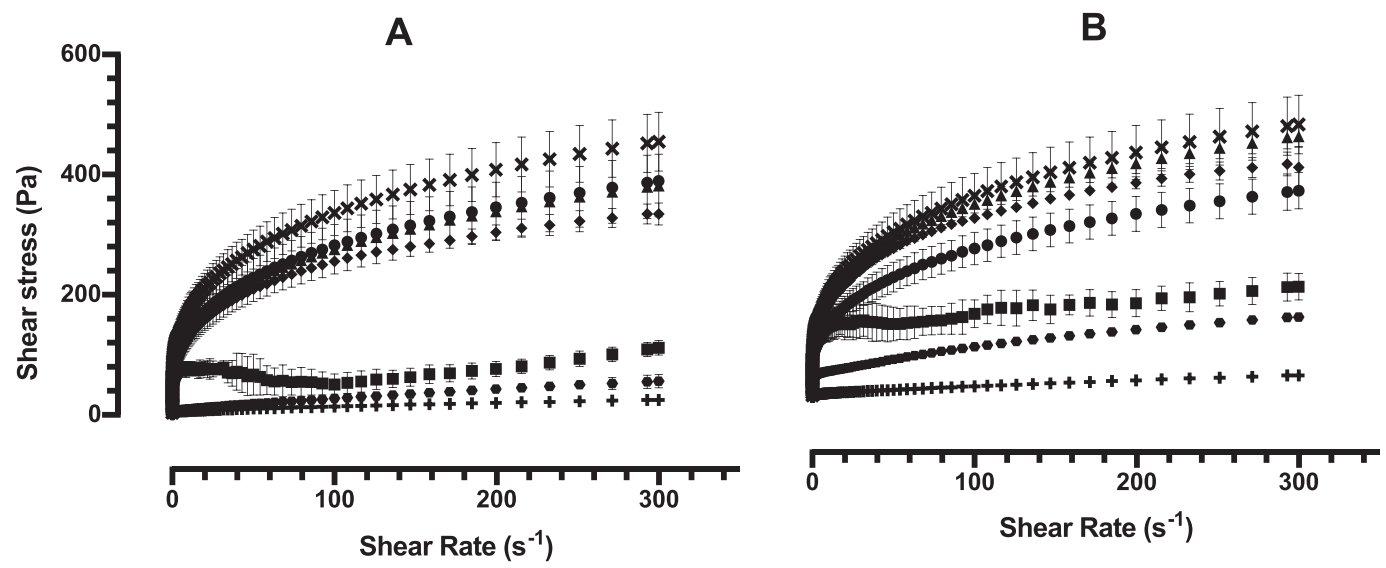

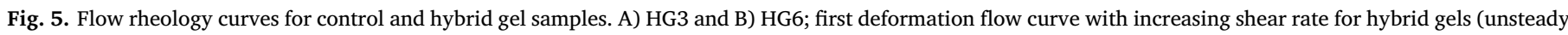
state) ( 1:99; $\mathbf{A}$ 5:95; 10:90; 20:80; 50:50; + control OG; × control AL).

Flow curves show how the use of OG6 influenced HG deformation behaviour (Fig. 5). HG6 samples recorded wider shear stress values (for the same shear-rate) between the different ratios. The HG6 with 50:50 and 20:80 ratios revealed higher values of shear stress than HG3. The tendency for HGs with higher ratios of oleogel to present lower stress values when shear rate increases, is in agreement with the morphological and microscopy analyses showed in Fig. 2 and Fig. 3. This may be a result of the effect imparted by the addition of larger proportions of oleogels and the consequent inability to incorporate successfully this phase within the hydrogel matrix. The shear-thinning effect attributed to alginate hydrogels was observed for all HG samples. The effect of the increase in oleogelator concentration was also visible as larger "stress overshoots" in the flow curves (first shear rate increasing- unsteady state) were visible for HG3 20:80, HG6 20:80 and HG6 50:50. This stress overshoot in flow curves can be indicative of a structure that was destroyed by the shear movement that was induced in the rheometer. A more well-built network is identified in this case, as this peak of shear stress is associated with the force, that is necessary to break the structural elastic network inducing flow behaviour. This is a result of the breakage of the gel structure with increasing shear rate where irreversible deformation is found. This flow behaviour is depicted by the alignment of the particles in the gel matrix, reducing viscosity. The same level of overshoot is not evidenced for the HG3 50:50 (Fig. 5A). As seen from the first flow curve, HG6 samples do not flow so easily, even after the applied shear. After the second shear rate sweep the results do not vary (steady state), and the only main observation is the decrease of the overshoot for the less organized samples; i.e. HG3 and HG6 with ratios of 50:50 and 20:80 (results not shown).

\section{Conclusions}

Hybrid gels produced with different oleogel and hydrogel ratios result in gels with diverse rheological and textural behaviors. The absence of polymorphic response from the XRD tests for the HGs showed the prevalence of the alginate fingerprint, as the waxes' crystal arrangement was not seen in the HGs results after the inclusion of oleogel in the hydrogel matrix. Different oleogelator concentrations (HG3 vs HG6) do not produce differences in terms of texture-related parameters (i.e. firmness, spreadability and adhesivity), due to the strong shear that is applied during HG development. However, after increasing the oleogel fraction from $10 \%$ upwards (within the same type of hybrid gel; e.g. HG3) the registered textural results were significantly different. The exhibited spreadability and adhesivity values, varied in opposite ways with the incorporation of larger oleogel quantities. As firmness decreases, the work of shear (spreadability) follows the same trend and consequently the adhesivity of the samples decreased. Gel-like behaviour $\left(G^{\prime}>G^{\prime}\right)$ was identified for all HGs, within the entire range of applied frequencies. However, high deformation rheology and textural experiments showed the tendency for hybrid gel samples to demonstrate lower values of such parameters alongside the increase of the oleogel ratio. The oscillatory rheometry results pointed out that HG3 and HG6 samples with 20:80 ratio are the ones with a higher viscoelastic response, that can be associated to the gel particle structure evidenced in polarized microscopy, where the gelled particles are bigger in size but still part of an interconnected structure (despite samples with increased oleogel ratio showed visible disaggregation under the microscope). Texture properties can be changed by varying oleogel and hydrogel ratios in HG formulation. Tailoring ability is evidenced and it is an advantage for these structures in order to use hybrid gels in applications where gel strength and shear capability are crucial parameters.

\section{Acknowledgments}

The authors acknowledge the Project RECI/BBB-EBI/0179/2012 (FCOMP-01-0124-FEDER-027462). Artur Martins is recipient of a fellowship supported by a doctoral advanced training (call NORTE-692015-15) funded by the European Social Fund under the scope of Norte2020 - Programa Operacional Regional do Norte. Cunha thanks CNPq (307168/2016-6) for the productivity grant. This research was supported by Norte Regional Operational Program 2014-2020 (Norte2020) through the European Regional Development Fund (ERDF) Nanotechnology based functional solutions (NORTE-01-0145-FEDER000019).

\section{Appendix A. Supplementary data}

Supplementary data to this article can be found online at https:// doi.org/10.1016/j.foodres.2018.10.019.

\section{References}

Cakmakc1 Gundogdu, E., \& Kavaz, A. (2008). Brighteners as a food additive. Academic Food. 6. Academic Food (pp. 17-22).

Cerqueira, M. A., Fasolin, L. H., Picone, C. S. F., Pastrana, L. M., Cunha, R. L., \& Vicente, A. A. (2017). Structural and mechanical properties of organogels: Role of oil and gelator molecular structure. Food Research International, 96, 161-170. https://doi. org/10.1016/j.foodres.2017.03.021.

Chung, C., Degner, B., Decker, E. A., \& McClements, D. J. (2013). Oil-filled hydrogel particles for reduced-fat food applications: Fabrication, characterization, and properties. Innovative Food Science \& Emerging Technologies, 20, 324-334. https://doi.org/ 10.1016/j.ifset.2013.08.006.

Ergun, R., Thomson, B. S., \& Huebner-Keese, B. (2016). Dow Global Technologies LLC, US Pat., 20160021898A1, 2016.

Esposito, C. L., Kirilov, P., \& Roullin, V. G. (2018). Organogels, promising drug delivery 
systems: an update of state-of-the-art and recent applications. Journal of Controlled Release, 271, 1-20. https://doi.org/10.1016/j.jconrel.2017.12.019.

Kamboj, S., \& Rana, V. (2014). Physicochemical, rheological and antioxidant potential of corn fiber gum. Food Hydrocolloids, 39, 1-9. https://doi.org/10.1016/j.foodhyd. 2013.12.015.

Li, Y., Jia, H., Pan, F., Jiang, Z., \& Cheng, Q. (2012). Enhanced anti-swelling property and dehumidification performance by sodium alginate-poly(vinyl alcohol)/polysulfone composite hollow fiber membranes. Journal of Membrane Science, 407-408, 211-220 https://doi.org/10.1016/j.memsci.2012.03.049.

Lin, C.-C., \& Metters, A. T. (2006). Hydrogels in controlled release formulations: Network design and mathematical modeling. Advanced Drug Delivery Reviews, 58(12-13), 1379-1408. https://doi.org/10.1016/j.addr.2006.09.004.

Lupi, F. R., Gentile, L., Gabriele, D., Mazzulla, S., Baldino, N., \& de Cindio, B. (2015). Olive oil and hyperthermal water bigels for cosmetic uses. Journal of Colloid and Interface Science, 459, 70-78. https://doi.org/10.1016/j.jcis.2015.08.013.

Martins, A. J., Cerqueira, M. A., Fasolin, L. H., Cunha, R. L., \& Vicente, A. A. (2016). Beeswax organogels: Influence of gelator concentration and oil type in the gelation process. Food Research International, 84, 170-179. https://doi.org/10.1016/j.foodres. 2016.03.035.

Martins, A. J., Vicente, A. A., Cunha, R. L., \& Cerqueira, M. A. (2018). Edible oleogels: an opportunity for fat replacement in foods. Food Funct. 9(2), 758-773. https://doi.org/ 10.1039/C7FO01641G.

Matalanis, A., \& McClements, D. J. (2013). Hydrogel microspheres for encapsulation of lipophilic components: Optimization of fabrication \& performance. Food Hydrocolloids, 31(1), 15-25. https://doi.org/10.1016/j.foodhyd.2012.09.012.

Mert, B., \& Demirkesen, I. (2016). Reducing saturated fat with oleogel/shortening blends in a baked product. Food Chemistry, 199 https://doi.org/10.1016/j.foodchem.2015. 12.087.

Mun, S., Kim, Y. R., \& McClements, D. J. (2015). Control of??-carotene bioaccessibility using starch-based filled hydrogels. Food Chemistry, 173, 454-461. https://doi.org/ 10.1016/j.foodchem.2014.10.053.

Mura, P., Faucci, M. T., Bramanti, G., \& Corti, P. (2000). Evaluation of transcutol as a clonazepam transdermal permeation enhancer from hydrophilic gel formulations. European Journal of Pharmaceutical Sciences, 9(4), 365-372. https://doi.org/10.1016/ S0928-0987(99)00075-5.

Öğütcü, M., \& Yılmaz, E. (2014). Oleogels of virgin olive oil with carnauba wax and monoglyceride as spreadable products. Grasas Y Aceites, 65(3), e040 https://doi.org/ 10.3989/gya.0349141.

Patel, A. R. (2015). Natural Waxes as Oil Structurants. 15-27. https://doi.org/10.1007/ 978-3-319-19138-6_2.

Patel, A. R., \& Dewettinck, K. (2016). Edible oil structuring: an overview and recent updates. Food Funct. https://doi.org/10.1039/C5FO01006C.

Peppas, N. A., Bures, P., Leobandung, W., \& Ichikawa, H. (2000). Hydrogels in pharmaceutical formulations. European Journal of Pharmaceutics and Biopharmaceutics, 50(1), 27-46. https://doi.org/10.1016/S0939-6411(00)00090-4.

Rehman, K., Mohd Amin, M. C. I., \& Zulfakar, M. H. (2014). Development and Physical
Characterization of Polymer-Fish Oil Bigel (Hydrogel/Oleogel) System as a Transdermal Drug Delivery Vehicle. Journal of Oleo Science, 63(10), 961-970. https://doi.org/10.5650/jos.ess14101.

Rogers, M. A., Strober, T., Bot, A., Toro-Vazquez, J. F., Stortz, T., \& Marangoni, A. G. (2014). Edible oleogels in molecular gastronomy. International Journal of Gastronomy and Food Science, 2(1), 22-31. https://doi.org/10.1016/j.ijgfs.2014.05.001.

Sagiri, S. S., Singh, V. K., Kulanthaivel, S., Banerjee, I., Basak, P., Battachrya, M. K., \& Pal, K. (2015). Stearate organogel-gelatin hydrogel based bigels: physicochemical, thermal, mechanical characterizations and in vitro drug delivery applications. $J$ Mech Behav Biomed Mater, 43, 1-17. https://doi.org/10.1016/j.jmbbm.2014.11.026.

Sanders Iii, C. T., DeMasie, C. L., Kerr, W. L., Hargrove, J. L., Pegg, R. B., \& Swanson, R. B. (2014). Peanut skins-fortified peanut butters: Effects on consumer acceptability and quality characteristics. LWT - Food Science and Technology, 59(1), 222-228 https:// doi.org/10.1016/j.lwt.2014.04.001.

Singh, V. K., Banerjee, I., Agarwal, T., Pramanik, K., Bhattacharya, M. K., \& Pal, K. (2014). Guar gum and sesame oil based novel bigels for controlled drug delivery. Colloids and Surfaces B: Biointerfaces, 123, 582-592. https://doi.org/10.1016/j.colsurfb.2014.09. 056.

Sloan, E. (2015). The Top Ten Functional Food Trends. Food Technology Magazine, 64.

Soltani, S., \& Madadlou, A. (2016). Two-step sequential cross-linking of sugar beet pectin for transforming zein nanoparticle-based Pickering emulsions to emulgels. Carbohydrate Polymers, 136, 738-743. https://doi.org/10.1016/j.carbpol.2015.09. 100.

Soradech, S., Petchtubtim, I., Thongdon-A, J., \& Muangman, T. (2016). Development of wax-incorporated emulsion gel beads for the encapsulation and intragastric floating delivery of the active antioxidant from Tamarindus indica L. Molecules.. https://doi. org $/ 10.3390 /$ molecules 21030380 .

Utrilla, M. C., García Ruiz, A., \& Soriano, A. (2014). Effect of partial reduction of pork meat on the physicochemical and sensory quality of dry ripened sausages: Development of a healthy venison salchichon. Meat Science, 98(4), 785-791. https:// doi.org/10.1016/j.meatsci.2014.07.027.

Wakhet, S., Singh, V. K., Sahoo, S., Sagiri, S. S., Kulanthaivel, S., Bhattacharya, M. K., \& Pal, K. (2015). Characterization of gelatin-agar based phase separated hydrogel, emulgel and bigel: a comparative study. Journal of Materials Science: Materials in Medicine, 26(2), 118 https://doi.org/10.1007/s10856-015-5434-2.

Wang, F. C., Gravelle, A. J., Blake, A. I., \& Marangoni, A. G. (2016). Novel trans fat replacement strategies. Current Opinion in Food Science, 7, 27-34. https://doi.org/10. 1016/j.cofs.2015.08.006

Yan, C., \& Pochan, D. J. (2010). Rheological properties of peptide-based hydrogels for biomedical and other applications. Chemical Society Reviews, 39(9), 3528 https://doi. org/10.1039/b919449p.

Zheng, B., Zhang, Z., Chen, F., Luo, X., \& McClements, D. J. (2017). Impact of delivery system type on curcumin stability: Comparison of curcumin degradation in aqueous solutions, emulsions, and hydrogel beads. Food Hydrocolloids, 71(Supplement C), 187-197. https://doi.org/10.1016/j.foodhyd.2017.05.022. 